\title{
TRONCO RADICULAR E SUA IMPORTÂNCIA NO ESTABELECIMENTO E PROGRESSÃO DA DOENÇA PERIODONTAL. UMA DISCUSSÃO BASEADA EM EVIDÊNCIAS.
}

Root trunk and its importance in the progression of periodontal disease. A discussion based on evidences.

Caroline Montez, Cirurgiã-dentista e Mestranda em Clínica Odontológica pela Universidade Federal Fluminense

Eliane dos Santos Porto Barboza, Mestre e Doutora em Periodontia pela Universidade de Boston e Professora Titular da Faculdade de Odontologia da Universidade Federal Fluminense

Categoria do trabalho: Artigo de revisão

Palavras-chave: tronco radicular, área de pré-furca, doença periodontal, envolvimento de furca.

Keywords: root trunk, pre furcation área, periodontal disease, furcation involvement.

Endereço para correspondência: Rua Mário Santos Braga, 28 - Centro - Niterói/RJ;

CEP:24020-140; Telefone: (21) 97980-8811

e-mail: elianeporto.uff@gmail.com 


\title{
RESUMO
}

Objetivo: A lesão de furca é definida como a reabsorção óssea patológica na área anatômica onde as raízes de dentes multirradiculares divergem. Os molares são os dentes mais afetados pela doença periodontal e a maior taxa de mortalidade desses dentes pode ser explicada pela morfologia que favorece o envolvimento de furca. Portanto, o dentista deve conhecer a anatomia da furca para diagnosticar precocemente seu envolvimento e tratar com precisão. O objetivo deste estudo é discutir, embasado em evidências científicas, a importância do tronco radicular no estabelecimento e progressão da doença periodontal. Materiais e métodos: uma revisão da literatura foi realizada na base de dados PubMed utilizando os termos tronco radicular, área de pré-furca, doença periodontal, envolvimento de furca em inglês. Resultados: Quarenta e três estudos foram selecionados e sete estudos foram incluídos nesta revisão. $O$ tronco radicular na superfície lingual é maior do que na vestibular em molares inferiores. A área de pré-furca foi encontrada em todos os molares estudados. Os pré-molares superiores também apresentam área de pré-furca e $37 \%$ são bifurcados. A altura do tronco radicular e a concavidade da pré-furca interferem no tratamento. Conclusão: As características morfológicas do tronco radicular e a profundidade e largura da área de pré-furca podem favorecer o estabelecimento e progressão da doença periodontal.

Palavras-chave: tronco radicular, área de pré-furca, doença periodontal, envolvimento de furca.

\begin{abstract}
Objective: The furcation lesion is defined as pathological bone resorption in the anatomical area where the roots of multiradicular teeth differ. Molars are the teeth most affected by periodontal disease and the higher mortality rate of these teeth can be explained by the morphology that favors the furcation involvement. In this way, the dentist must know the anatomy of the furca to diagnose its involvement early and to treat with precision. The aim of this study is to discuss, based cientific evidences, the importance of the root trunk for the establishment and development of the periodontal disease. Material and Methods: A literature review was carried out in the PubMed database using the terms root trunk, pre furcation área, periodontal disease, furcation involvement in english. Results: Forty-three studies were selected and sextuplets were found at the time of the review. The root trunk at the lingual surface is larger than the buccal surface in lower molars. A pre-furcation area was found in all molars studied. Upper premolars also have a pre-furcation area and $37 \%$ are bifurcated. The height of the root trunk and the concavity of the pre-furcation area interfere in the treatment. Conclusion: Root trunks and concavity of the pre furcation area may favor the stablishment and progression of periodontal disease.
\end{abstract}

Keywords: root trunk, pre furcation área, periodontal disease, furcation involvement. 


\section{INTRODUÇÃO}

A lesão de furca é a reabsorção óssea patológica na área anatômica onde as raízes de dentes multirradiculares divergem (AAP, 2001). A morfologia da lesão de furca favorece a retenção de biofilme, o que dificulta o controle profissional e pessoal da placa dentária e afeta positivamente a patogênese da destruição periodontal (BARBOZA Jr. et al., 2014; LARATO, 1975) .

O tratamento escolhido para o envolvimento de furca depende do conhecimento da sua morfologia para diagnosticar corretamente. A medida do tronco radicular (TR), que é a distância entre a junção cemento-esmalte e a entrada da furca (figura 1), e a característica anatômica conhecida como área de pré-furca (APF), que é a concavidade que se estende verticalmente tanto na vestibular quanto na lingual, são fundamentais para compreender a prevenção, o estabelecimento, a progressão, e o tratamento da doença periodontal (LU, 1992; ROUSSA, 1998).

O objetivo deste trabalho foi discutir, embasado em evidências científicas, a importância da morfologia do tronco radicular no estabelecimento e desenvolvimento da doença periodontal.

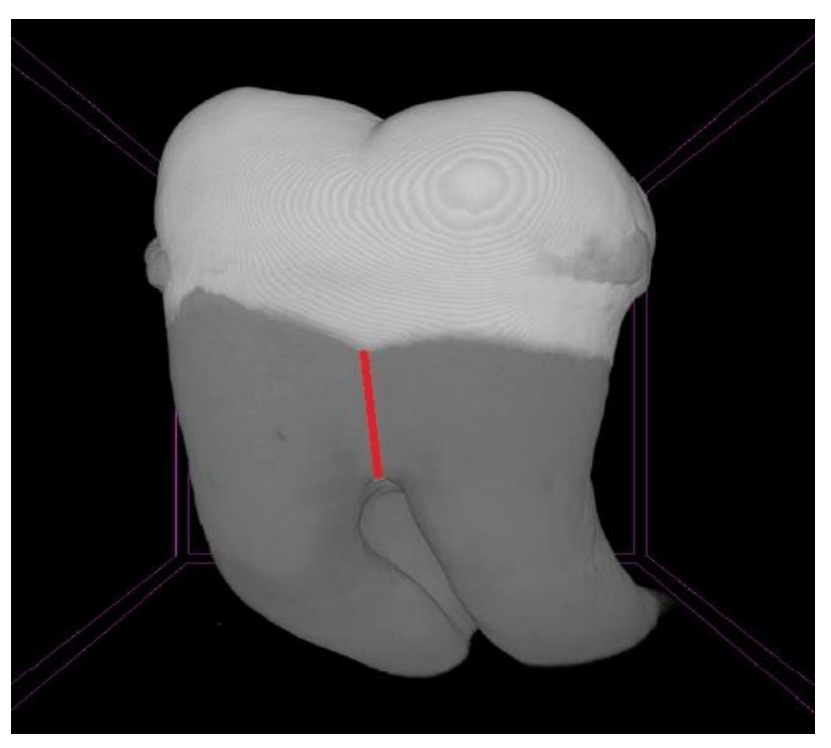

Figura 1- Imagem obtida por microtomografia digital, note o comprimento do tronco radicular em vermelho. 


\section{MATERIAL E MÉTODOS}

O presente estudo fez uma revisão de literatura atual elaborada através da coletânea de artigos científicos com texto completo disponível por meio de um levantamento bibliográfico na base de dados online PubMed. Ferramentas de busca de dados avançada e as palavras-chave "root trunk", "pre furcation area", "periodontal disease", "furcation involvement" foram utilizadas. Os termos concavidade ou sulco presente no tronco radicular ou concavidade coronal à furca foram atualizados para o termo "área de pré-furca", com base em Barboza Jr. et al. 2014.

\section{Critérios de inclusão}

Ensaios clínicos randomizados e estudos in-vitro publicados em inglês com texto completo disponível, que relataram as características morfológicas do tronco radicular e da área préfurca e suas relevâncias clínicas.

\section{Critérios de exclusão}

Artigos que discutiram o diagnóstico e o tratamento das lesões de furca, sem fazer relação com as características anatômicas do tronco radicular. Após análise de todos os textos, sete artigos foram separados e incluídos nessa revisão de literatura (tabela 1).

Tabela 1- As características dos estudos incluídos e seus desfechos primários e secundários.

\begin{tabular}{|c|c|c|c|c|}
\hline Estudo & $\begin{array}{l}\text { Desenho do } \\
\text { Estudo }\end{array}$ & $\begin{array}{l}\text { Amostra da } \\
\text { Pesquisa }\end{array}$ & Tronco Radicular (TR) & Área de Pré-furca (APF) \\
\hline $\begin{array}{l}\text { Joseph et al., } \\
1996\end{array}$ & In vitro & $\begin{array}{l}100 \text { primeiros } \\
\text { pré-molares } \\
\text { superiores }\end{array}$ & $\begin{array}{l}\text { Comprimento médio foi } \\
7,9 \mathrm{~mm} \text { na mesial e } 7,6 \mathrm{~mm} \\
\text { na distal. }\end{array}$ & $\begin{array}{l}\text { A APF é mais profunda na } \\
\text { mesial }(0,61 \mathrm{~mm}) \text { que na } \\
\text { distal }(0,30 \mathrm{~mm}) \text { dos pré- } \\
\text { molares. }\end{array}$ \\
\hline Roussa, 1998 & In vitro & 60 molares & $\begin{array}{l}\text { A média de comprimento } \\
\text { nos } 1^{\text {os }} \text { molares inferiores } \\
\text { foi } 2,80 \mathrm{~mm} \text { na vestibular e } \\
3,50 \mathrm{~mm} \text { na lingual; nos } 2^{\text {os }} \\
\text { molares inferiores foi de } \\
3,40 \mathrm{~mm} \text { na vestibular e } \\
3,70 \mathrm{~mm} \text { na lingual. Nos } \\
\text { molares superiores, a }\end{array}$ & $\begin{array}{l}\text { A média de profundidade } \\
\text { da APF encontrada foi de } \\
1,01 \mathrm{~mm} \text { na vestibular e } \\
0,88 \mathrm{~mm} \text { na lingual dos } 1^{\text {os }} \\
\text { molares inferiores; } \\
0 ., 78 \mathrm{~mm} \text { na vestibular e } \\
1,00 \mathrm{~mm} \text { na lingual dos } 2^{\text {os }} \\
\text { molares inferiores; Nos }\end{array}$ \\
\hline
\end{tabular}

REVISTA FLUMINENSE DE ODONTOLOGIA - ANO XV - No 51 - Janeiro / Junho 2019 


\begin{tabular}{|c|c|c|c|c|}
\hline & & & $\begin{array}{l}\text { média encontrada nos } 1^{\text {os }} \\
\text { foi } 3,49 \mathrm{~mm} \text { na mesial, } \\
4,14 \mathrm{~mm} \text { na distal e } \\
3.46 \mathrm{~mm} \text { na vestibular; nos } \\
2^{\text {os }} \text { foi } 3,28 \mathrm{~mm} \text { na mesial, } \\
3,95 \mathrm{~mm} \text { na distal e } \\
3,34 \mathrm{~mm} \text { na vestibular. }\end{array}$ & $\begin{array}{l}\text { superiores, a média } \\
\text { encontrada nos } 1^{\text {os }} \text { foi } \\
0,65 \mathrm{~mm} \text { na mesial, } \\
0,52 \mathrm{~mm} \text { na distal e } \\
0,72 \mathrm{~mm} \text { na vestibular; nos } \\
2^{\text {os }} \text { foi } \\
0,61 \mathrm{~mm} \text { na mesial, } \\
0,29 \mathrm{~mm} \text { na distal e } \\
0.55 \pm 0.53 \mathrm{~mm} \text { na } \\
\text { vestibular. }\end{array}$ \\
\hline $\begin{array}{l}\text { De los Rios et } \\
\text { al., } 2002\end{array}$ & In vitro & $\begin{array}{l}100 \text { segundos } \\
\text { molares inferiores }\end{array}$ & $\begin{array}{l}\text { O comprimento médio do } \\
\text { TR de } 3,09 \mathrm{~mm} \text { na } \\
\text { vestibular e } 3,91 \mathrm{~mm} \text { na } \\
\text { lingual. }\end{array}$ & $\begin{array}{l}\text { Largura média da APF foi } \\
\text { de } 3,6 \mathrm{~mm} \text { na vestibular e } \\
3,3 \mathrm{~mm} \text { na lingual. } \\
\text { Comprimento médio da } \\
\text { APF foi de } 2,93 \mathrm{~mm} \text { na } \\
\text { vestibular e } 3,61 \mathrm{~mm} \text { na } \\
\text { lingual. A profundidade } \\
\text { média de APF foi de } \\
0,88 \mathrm{~mm} \text { na vestibular e } \\
0,77 \mathrm{~mm} \text { na lingual. }\end{array}$ \\
\hline $\begin{array}{l}\text { Villaça et al., } \\
2004\end{array}$ & Prospectivo & $\begin{array}{l}10 \text { participantes } \\
\text { com duas lesões } \\
\text { de furca similares } \\
\text { em molares } \\
\text { inferiores }\end{array}$ & $\begin{array}{l}\text { Não avaliou o } \\
\text { comprimento do TR, } \\
\text { calculou o resultado da } \\
\text { regeneração tecidual } \\
\text { guiada de acordo com o } \\
\text { comprimento de TR em } \\
\text { ganho ósseo vertical. }\end{array}$ & $\begin{array}{l}\text { Não avaliou a } \\
\text { profundidade da APF, } \\
\text { avaliou o resultado da } \\
\text { regeneração tecidual } \\
\text { guiada de acordo com a } \\
\text { profundidade da APF. }\end{array}$ \\
\hline $\begin{array}{l}\text { Do Vale et al., } \\
2009\end{array}$ & Prospectivo & $\begin{array}{l}23 \text { participantes } \\
\text { com periodontite } \\
\text { crônica }\end{array}$ & $\begin{array}{l}\text { A média radiográfica do } \\
\text { comprimento do TR } \\
\text { encontrada foi de } 3,92 \mathrm{~mm} \text {. }\end{array}$ & $\begin{array}{l}\text { Não avaliou a } \\
\text { profundidade da APF. }\end{array}$ \\
\hline $\begin{array}{l}\text { Marcaccini et } \\
\text { al., } 2012\end{array}$ & In vitro & $\begin{array}{l}233 \text { primeiros } \\
\text { molares inferiores }\end{array}$ & $\begin{array}{l}\text { O comprimento médio de } \\
\text { TR foi de } 1,37 \mathrm{~mm} \mathrm{na} \\
\text { vestibular e } 2,04 \mathrm{~mm} \text { na } \\
\text { lingual. }\end{array}$ & $\begin{array}{l}\text { Não avaliou a } \\
\text { profundidade da APF. }\end{array}$ \\
\hline $\begin{array}{l}\text { Barboza Jr et al., } \\
2014\end{array}$ & In vitro & $\begin{array}{l}96 \text { primeiros } \\
\text { molares inferiores }\end{array}$ & $\begin{array}{l}\text { Comprimento médio do } \\
\text { TR na vestibular foi }\end{array}$ & $\begin{array}{l}\text { Todos os dentes } \\
\text { apresentaram APF e as }\end{array}$ \\
\hline
\end{tabular}




\begin{tabular}{|l|l|l|l|l|}
\hline & & $3,07 \mathrm{~mm}$ e lingual $3,54 \mathrm{~mm}$. & $\begin{array}{l}\text { médias de profundidade } \\
\text { são de } 0,89 \mathrm{~mm} \text { na } \\
\text { vestibular e } 0,82 \mathrm{~mm} \text { na } \\
\text { lingual. }\end{array}$ \\
\hline
\end{tabular}

\section{RESULTADOS}

Um estudo anatômico feito com 60 molares extraídos identificou possíveis fatores morfológicos que podem afetar o diagnóstico, prognóstico e o manejo clínico no tratamento periodontal das lesões de furca. Todos os dentes analisados apresentaram APF e os dentes que exibiam projeção cervical de esmalte as áreas de pré-furca se apresentavam mais profundas (ROUSSA, 1998).

Um estudo realizado por Marcaccini et al. (2012) avaliou a dimensão das raízes na área da furca em 233 primeiros molares mandibulares permanentes por meio de microfotografias digitais. $\mathrm{O}$ tronco radicular da superfície vestibular encontrada foi significativamente menor que o da superfície lingual (MACACCINI et al., 2012). Outra pesquisa, estudou com instrumentos biométricos 96 primeiros molares mandibulares permanentes para analisar os perfis vestibulares e linguais da superfície da junção cemento esmalte à entrada da furca. $\mathrm{O}$ comprimento e a profundidade do tronco radicular e da área de pré-furca foram registrados. Os autores encontraram o comprimento médio do tronco radicular de $3,07 \mathrm{~mm}$ na vestibular e 3,57mm na lingual. A área de pré-furca foi encontrada em $100 \%$ dos dentes estudados e sua profundidade máxima encontrada foi de $4 \mathrm{~mm}$ apical à junção cemento-esmalte. A média da profundidade foi de $0,89 \mathrm{~mm}$ na vestibular e $0,82 \mathrm{~mm}$ na lingual (LARATO, 1975). O estudo mais recente nesta área avaliou microtomograficamente 30 primeiros molares inferiores e encontrou a média do tronco radicular de $2,49 \mathrm{~mm}$ na vestibular e $3,18 \mathrm{~mm}$ na lingual. As larguras médias da área de pré-furca, na junção cemento-esmalte, $1 \mathrm{~mm}$ e $2 \mathrm{~mm}$ em direção à entrada da furca foram $2,9 \mathrm{~mm}, 3,4 \mathrm{~mm}, 3,9 \mathrm{~mm}, 4,3 \mathrm{~mm}$, respectivamente, enquanto as profundidades médias foram $0,19 \mathrm{~mm}, 0,32 \mathrm{~mm}, 0,57 \mathrm{~mm}, 1,1 \mathrm{~mm}$, respectivamente.

Em 2002, um artigo avaliou o comprimento, largura e profundidade do tronco radicular de 100 segundos molares inferiores e encontrou que o comprimento do tronco radicular foi de $3,09 \mathrm{~mm}$ na vestibular e $3,91 \mathrm{~mm}$ na lingual. A profundidade média da área de pré-furca foi de $2,93 \mathrm{~mm}$ na vestibular e $3,61 \mathrm{~mm}$ na lingual. O trabalho também revelou que existe área de pré-furca nos segundos molares inferiores cuja profundidade e largura foram maiores na superfície vestibular e o comprimento foi maior na superfície lingual (DE LOS RIOS et al., 2002).

Pioneiramente, um estudo avaliou o tamanho do tronco radicular, a concavidade do tronco radicular e os aspectos da furca de 100 primeiros pré-molares superiores. Os autores verificaram que $37 \%$ da amostra apresentava bifurcação, o comprimento mínimo do tronco 
radicular encontrado foi de 4,0mm e o máximo foi de $14,6 \mathrm{~mm}$, encontrando um comprimento médio de $7,6 \mathrm{~mm}$. A área de pré-furca foi encontrada no aspecto palatino da raiz vestibular em $62 \%$ dos dentes bifurcados. Além disso, concavidades foram encontradas tanto na mesial quanto na distal das raízes de 100\% dos dentes (JOSEPH et al., 1996).

Outras pesquisas relacionaram os fatores anatômicos do tronco radicular e da área de préfurca com o tratamento da lesão de furca. Um estudo prospectivo avaliou vinte e três pacientes com periodontite crônica que apresentavam envolvimento de furca classe II em molares inferiores e profundidade de sondagem maior ou igual a $5 \mathrm{~mm}$, antes e depois de receberem tratamento periodontal não-cirúrgico. Os autores avaliaram os parâmetros clínicos e os parâmetros radiográficos seis meses após o tratamento e encontraram relação entre morfologia e resposta ao tratamento não cirúrgico na altura da lesão, largura da furca no nível da crista alveolar e $2 \mathrm{~mm}$ apical ao fornix que podem ser indicadores de prognóstico. O nível ósseo aumentou em 1,08mm e a margem gengival em 2,74mm. Os autores avaliaram que quanto menor e mais estreita é a furca, melhores resultados são esperados (DO VALE et al., 2009). Em 2004, um estudo prospectivo avaliou o resultado de procedimentos regenerativos em lesões de furca em dentes que apresentavam áreas de préfurca no tronco radicular. Dez pacientes com dez pares de defeitos de furca classe II similares foram selecionados $\mathrm{e}$ os envolvimentos de furca foram divididos randomizadamente para metade receber enxerto com membrana expandida de politetrafluoroetileno (ePTFE) e a outra metade recebeu membrana de ePTFE modificada. $\mathrm{O}$ colar da membrana normal foi cortado em pedaços de $2 \mathrm{~mm}$ e posteriormente esses pedaços foram suturados com um colar de membrana. Após um ano, os autores identificaram que a membrana modificada apresentou melhores resultados na regeneração horizontal do defeito de furca classe II (VILAÇA et al., 2004).

\section{DISCUSSÃO}

O tronco radicular apresenta a relevância clínica de ser uma região anatômica com facilidade de reter biofilme e de predizer o prognóstico de desenvolvimento da doença periodontal de acordo com o seu comprimento (LARATO, 1975). O comprimento do tronco radicular é maior na lingual do que na vestibular e a média encontrada foi de 3,07mm na vestibular e 3,54mm na lingual em primeiros molares inferiores, enquanto os segundos molares inferiores apresentaram média de 3,09mm e 3,91mm, respectivamente (BARBOZA Jr et al., 2014; ROUSSA, 1998; MARCACCINI et al., 2012; DE LOS RIOS et al., 2002). Enquanto os pré-molares apresentaram uma média de 7,6mm sem distinguir diferença de comprimento entre vestibular e lingual (DO VALE, 2009). O estudo de Perminio et al. (2018) é o mais recente em relação ao comprimento do Tronco Radicular e avaliou através de microtomografia, que confere mais precisão métrica, o comprimento do TR de 30 primeiros molares inferiores, os resultados encontrados foi uma média de 2,49mm na vestibular e $3,18 \mathrm{~mm}$ na lingual. 
Recentemente, a nova classificação das doenças periodontais relatou a complexidade da periodontite (CATON et al., 2018). O estágio I apresentou profundidade de sondagem máxima de 4mm, enquanto os estágios II e III, $5 \mathrm{~mm}$ e mais de $6 \mathrm{~mm}$, respectivamente. Isso indica que já no estágio I, os molares podem apresentar envolvimento de furca tanto na vestibular quanto na lingual. Dessa forma, quanto maior o comprimento do tronco radicular, melhor o prognóstico em relação ao estabelecimento da lesão de furca. No entanto, ao apresentar envolvimento de furca, o tronco radicular longo dificulta o acesso operatório e o prognóstico se torna desfavorável.

O termo "área de pré-furca" foi inserido na literatura em 2014 para se referir a concavidade presente no tronco radicular de todos os dentes multirradiculares (BARBOZA Jr et al., 2014). No entanto, outros autores também tinham relatado a presença de uma concavidade coronal a entrada da furca (ROUSSA, 1998; MARCACCINI et al., 2012; DE LOS RIOS et al., 2002; JOSEPH et al., 1996). A média de profundidade dessa concavidade encontrada foi de $0,82 \mathrm{~mm}$ na lingual e $0,89 \mathrm{~mm}$ na vestibular (BARBOZA Jr et al., 2014). Além disso, Roussa (1998) identificou que dentes que possuem projeção cervical de esmalte tinham concavidades na raiz mais profundas quando comparadas aos dentes sem projeção. Perminio et al. (2018) relatou que a junção cemento-esmalte coincidiu com a área de pré-furca em $33,3 \%$ dos dentes estudados.

Os autores relataram que quanto maior a profundidade da APF, maior o envolvimento de furca devido à retenção de biofilme e pela dificuldade de acesso dos instrumentais para fazer o debridamento adequado, afetando diretamente o tratamento da lesão (BARBOZA Jr et al., 2014; ROUSSA, 1998; DO VALE et al., 2009). Além disso, a coincidência da APF a partir da junção cemento esmalte em $1 / 3$ das superfícies estudadas representa um maior fator de risco para o desenvolvimento precoce de lesões de furca (BARBOZA Jr et al., 2014; DE LOS RIOS et al., 2002). Dessa forma, tanto a região em que essa concavidade se encontra quanto a sua profundidade interferem no estabelecimento, progressão e prognóstico da doença periodontal.

Os fatores anatômicos do tronco radicular são fatores locais que influenciam diretamente no tratamento (DO VALE, 2009; VILAÇA, 2004).O comprimento do tronco radicular e a largura e profundidade da concavidade da área de pré-furca podem influenciar na terapia periodontal não cirúrgica de lesões de furca mandibulares classe II. O prognóstico esperado é melhor para dentes que apresentam troncos radiculares menores e mais estreitos. Mesmo que os resultados clínicos apresentem alterações positivas em relação ao sangramento à sondagem e nível ósseo, imagens radiográficas devem ser usadas para uma avaliação completa após o tratamento (DO VALE, 2009).

A área de pré-furca presente no tronco radicular influencia negativamente a regeneração de lesões de furca classe II por dificultar a adaptação da membrana. Por isso, Villaça et al. (2004) encontrou resultados melhores quando usou uma membrana de ePTFE modificada. 
COPYRIGHT C 2019 INTERNATIONAL JOURNAL OF SCIENCE DENTISTRY | AVAILABLE ONLINE

Os autores indicaram modificações no design da membrana para promover uma adaptação mais íntima à concavidade do tronco radicular e bloquear efeitos adversos.

\section{CONCLUSÃO}

Com base na literatura discutida, pode ser concluído que o tronco radicular e a área de préfurca influenciam no estabelecimento e progressão da doença periodontal, devido à retenção de biofilme e a dificuldade de acesso da região para o tratamento. Portanto, o conhecimento das características anatômicas é fundamental para a escolha adequada do tratamento e para o prognóstico das lesões de furca.

\section{REFERÊNCIAS BIBLIOGRÁFICAS}

1. American Academy of Periodontology. Glossary of periodontal terms. 4th ed. Chicago: American Academy of Periodontology 2001.

2. Barboza CA Jr., Rodrigues DM, Petersen R, Tristão GC, Barboza EP. Biometric study of the prefurcation area of human mandibular first molars. Int J Periodontics Restorative Dent 2014;34(6):857-61.

3. Larato DC. Some anatomical factors related to furcation involvements. J of Periodontol 1975; 46(10):608-609.

4. Lu H-KJ. Topographical characteristics of root trunk length related to guided tissue regeneration. J Periodontol 1992;63: 215-219.

5. Roussa, E. Anatomic Characteristics of the Furcation and Root Surfaces of Molar Teeth and Their Significance in the Clinical Management of Marginal Periodontitis. Clin Anat.1998;11:177-186.

6. Marcaccini AM, Pavanelo Â, Nogueira AVB, Souza JACD, Porciúncula HF, Cirelli JA. Morphometric study of the root anatomy in furcation area of mandibular first molars. Journal of Applied Oral Science. 2012; 20(1):76-81.

7. De los Rios CM, Pustiglioni FE, Romito GA. Biometric study of the width, length and depth of the root trunk groove of human lower second molars. Pesqui Odontol Bras. 2002;16:26-30.

8. Joseph I, Varma BRR, Bhat KM. Clinical significance of furcation anatomy of the maxillary first premolar: a biometric study on extracted teeth. J of periodontol. 1996; 67(4): 386-389.

9. Do Vale HF, Ribeiro ÉDP, Bittencourt S, Nociti FH Jr, Sallum EA, Casati MZ. Radiographic characteristics of furcation involvements in mandibular molars as prognostic indicators of healing after nonsurgical periodontal therapy. The $\mathrm{J}$ of the American Dent Associat 2009;140(4):434-440.

10. Villaça JH, Rodrigues DC, Novaes AB Jr, Taba M Jr, Souza SL, Grisi MF. Root trunk concavities as a risk factor for regenerative procedures of class II furcation lesions in humans. J of periodontol 2004;75(11):1493-1499. 
11. Perminio DJD. Micro Tomographic Analysis of the Root Trunk and Pre-furcation Area of the First Mandibular Molars. Tese (Doutorado em Odontologia) - Faculdade de Odontologia, Universidade Federal Fluminense. Rio de Janeiro. 2018.

12. Caton JG, Armitage G, Berglundh T,Chapple IL, Jepsen SS, Kornman K et al. A new classification scheme for periodontal and peri-implant diseases and conditions Introduction and key changes from the 1999 classification. J Clin Periodontol. 2018;45:1-8. 